\title{
Electrospinned bioactive glass/nano hydroxyapatite reinforced polycaprolactone GTR/GBR membranes in periodontics- A review
}

\author{
Vishnu J. S., 1,*, Aneesh Alim², Dilshi Ishaan², Basim Burhan K. B. ${ }^{4}$, Aswathy Krishna $\mathrm{S}^{5}$ \\ ${ }^{1,2}$ Senior Lecturer, ${ }^{3}$ Periodontist, Private Practitioner, ${ }^{4}$ Surgeon, ${ }^{1,2,4}$ Dept. of Periodontics, PMS College of Dental Science \& \\ Research, Thiruvananthapuram, Kerala, ${ }^{5}$ PG Student, Amrita School of Dentistry, Cochin, Kerala, India
}

*Corresponding Author:

Email: drvishnu731@gmail.com

\begin{abstract}
Periodontitis, a chronic inflammatory infectious disease triggered by an interaction between microbial biofilm and host response leading to gingival bleeding, connective tissue destruction, alveolar bone loss and finally causing tooth loss. Periodontal regeneration or restoration is the ultimate goal of any periodontal treatment. This can be achieved by GTR/GBR [Guided Tissue Regeneration/Guided Bone Regeneration] membranes along with bone replacement grafts. With the evolution of Tissue engineering and different generation of GTR/GBR nanofibrous membranes, periodontal restoration or regeneration can be easily and rapidly achieved . Nanofibrous GTR/GBR Membranes can be fabricated using natural or synthetic polymers by different techniques such as Phase separation, Self assembly and Electrospinning [E-spinning]. Of these, E-spinning is the most widely studied technique and also seems to exhibit the most promising results for Tissue Engineering[TE] applications . This review addresses the Electrospinning method of GTR/GBR membrane fabrication using Bioactive Glass [BG]/Nano Hydroxyapatite[nHAP] reinforced polycaprolactone polymer and its advantages and disadvantages.
\end{abstract}

Keywords: Electrospinning, Guided Tissue Regeneration, Periodontal Regeneration.

\section{Introduction}

Periodontitis, which is bacterially induced, can be defined as a chronic inflammatory disease initiated by dental plaque biofilm and perpetuated by a deregulated immune response usually accompanied by gingivitis resulting in irreversible destruction of the supporting tissues surrounding the tooth, including the periodontal ligament, cementum and the alveolar bone finally causing tooth loss. ${ }^{1}$ The goals of periodontal therapy are to cure the disease, to prevent disease recurrence, that is, maintain periodontal health and to restore periodontal tissues lost through the disease. ${ }^{2}$ Conventional periodontal surgical treatment modalities (surgical debridement and resective procedures) have been established as effective means of treating periodontal disease and arresting its progression. These methods typically heal by repair, with a combination of connective tissue adhesion/ attachment or formation of a long junctional epithelium. ${ }^{3}$ To obtain good stability and predictability after therapy, periodontal regeneration of destroyed tissue, which is characterized by de novo formation of cementum, a functionally organized PDL, alveolar bone, and gingiva, is desirable. The desire to induce the complete regeneration of periodontal tissue has inspired the introduction of Guided Tissue Regeneration technique. ${ }^{4}$

The term "Guided Tissue Regeneration (GTR)" was given by Gottlow in 1986. The 1996 World Workshop in Periodontics defined GTR as "procedures attempting to regenerate lost periodontal structures through differential tissue responses". The principle of GTR was based on Melchers hypothesis [1976] which states that certain cell populations residing in the periodontium have the potential to create new cementum, alveolar bone and PDL, when they are provided the opportunity to populate the periodontal wound. ${ }^{5}$ GTR employs a barrier membrane around the periodontal defect to prevent epithelial downgrowth and fibroblast transgrowth into the wound space, thereby maintaining a space for true periodontal tissue regeneration. The barrier membranes used for GTR can be broadly divided into three generations of membranes.

The first generation of barrier membranes were non resorbable membranes. The second generation of barrier membranes was designed to be resorbable to avoid the need for surgical removal. Third generation barrier membranes are developed based on the concept of Tissue Engineering [TE]. TE is a multi-disciplinary field, which aims to apply innovative biomaterials to replace or restore ill or damaged tissues of the human body, such as skin and bones. ${ }^{7}$ The triad for conventional cell-based TE involves cells, signaling molecules, and scaffold/supporting matrices. In this triad, the role of the scaffold is the "niche" of cells, and facilitates the attachment, migration, proliferation, and three-dimensional (3D) spatial organization of the cell population that defines the shape of the tissue that needs regeneration. ${ }^{8}$ Third-generation membranes have evolved, which not only act as barriers but also as delivery devices to release specific agents such as bioactive materials ,antibiotics, growth factors, adhesion factors, etc., at the wound site on a time or need basis in order to orchestrate and direct natural wound healing in a better way. 
Briefly they may be considered into the following sub divisions:

1. Barrier membranes with Antimicrobial activity

2. Barrier membranes with Bioactive materials

3. Barrier membranes with Growth Factor release

A number of novel approaches have been developed for the fabrication of biomaterial-based 3D scaffolds. Currently, some of the most promising scaffolding materials for application in bone tissue engineering are Hydroxyapatites[HA], Bioactive glasses[BG] and related biodegradable polymer materials(e.g. PCL, PGA etc). These scaffolds are highly porous, 3D structures exhibiting tailored porosity, pore size and interconnectivity. ${ }^{9}$ There is a high number of polymer bioceramic composite membrane manufacturing techniques, such as solvent casting, particulate leaching, Three dimensional printing, Thermally induced phase separation (TIPS). The development of nanofibers has enhanced the scope for fabricating bioactive membranes that can potentially mimic the architecture of natural human tissue at the nanometer scale. The high surface area to volume ratio of the nanofibers combined with their microporous structure favors cell adhesion, proliferation, migration, and differentiation, all of which are highly desired properties for tissue engineering applications. Therefore, current research in this area is driven towards the fabrication, characterization, and applications of nanofibrous systems for TE. ${ }^{10}$ Phase separation, Self assembly and Electrospinning [Espinning] are the three techniques available for fabricating nano structured fibers with variable morphological characters. ${ }^{11}$

\section{Review of Literature}

Regeneration of the reduced periodontium is the ideal goal in periodontal therapy. By definition, successful periodontal regeneration is the simultaneous regeneration of cementum, PDL, and alveolar bone, so that the form and function of the lost structures are restored. In 1976 Melcher formulated a hypothesis which suggested that, under physiological conditions, only cells from periodontal ligament can synthesize and secrete cementum to attach newly- synthesised collagen fibres to tooth. This hypothesis was experimentally and histologically verified by Karring et al. The necessity for exclusion of epithelial and connective tissue cells of the gingiva from the wound led to the development and application of Guided Tissue Regenertion (GTR) membranes. ${ }^{12}$ There are different generations of GTR Membranes .The most recent generation of GTR Membrane is based on degradable polymer Polyglycolide, polycaprolactone, polylactic acid incorporated with bioactive material such as hydroxy apatite, Bioactive glass, calcium sulphate etc. Among these biodegradable polymer, Polycaprolactone shown great promise as a barrier membrane for tissue engineering. ${ }^{13}$
Remya et al in $2013^{14}$ fabricated Nanohydroxyapatite Incorporated Electrospun Polycaprolactone / Polycaprolactone - Polyethyleneglycol Polycaprolactone blend Scaffold for Bone Tissue Engineering Applications. The study was a comparative evaluation of physical and biological properties of electrospun biodegradable fibrous scaffolds based on polycaprolactone (PCL) and its blend with polycaprolactone-polyethyleneglycol-polycaprolactone (CEC) with and without nanohydroxyapatite (nHAP) particles. The fiber morphology, porosity, surface wettability, and mechanical properties of electrospun PCL were distinctly influenced by the presence of both copolymer CEC and nHAP. The degradation in hydrolytic media affected both morphological and mechanical properties of the scaffolds and the tensile strength decreased by $58 \%$ for PCL, $83 \%$ for PCL/CEC, $36 \%$ for PCL/nHAP and $75 \%$ for PCL/CEC/nHAP in 90 days of PBS ageing. MTT assay using mouse fibroblast L929 cells proved all the scaffolds to be non-cytotoxic. These results reveaed that the potential of the cytocompatible PCL/CEC/nHAP scaffold for the fabrication of living bony constructs for tissue engineering applications.

Hassan et al in $2012^{15}$ prepared HA/PCL Scaffolds for tissue engineering. The wet slurry of HA was produced by mixing an acetone solution of calcium nitrate 4-hydrate with an aqueous solution of ammonium phosphate and ammonium carbonate with control $\mathrm{pH}$ of 11 . The nano-emulsion was kept in freezer about one day and after that was kept in freeze drying machine about three days to obtain dry HA powder with low degree of agglomeration. The nanoparticles were studied under scanning electron microscopy (SEM). The polycaprolactone (PCL) and hydroxyapatite/ polycaprolactone (HA/PCL) composite scaffolds were produced using thermally induced phase separation (TIPS) technique. The scaffolds were studied under SEM and it was observed that both types of scaffolds had porous structures. The pore sizes of HA/PCL scaffold was slightly decreased compared to PCL scaffold. The authors concluded that both PCL and HA/PCL scaffolds showed promises for bone tissue engineering application.

Park et al in $2011^{16}$ fabricated porous polycaprolactone/hydroxyapatite (PCL/HA) blend scaffolds using a 3D plotting system for bone tissue engineering. The authors designed and fabricated three types of scaffolds: those from polycaprolactone (PCL), those from PCL and hydroxyapatite (HA), and those from PCL/HA and with a shifted pattern structure (PCL/HA/SP scaffold). Shifted pattern structure was fabricated to increase the cell attachment/adhesion. The PCL/ HA/SP scaffold had a lower compressive modulus than PCL and PCL/HA scaffold. MTT assay and alkaline phosphatase activity results for the PCL/HA/SP scaffolds were significantly enhanced compared to the results for the PCL and PCL/HA scaffolds. According to their degree of cell 
proliferation/differentiation, the scaffolds were in the following order: PCL/HA/ SP> PCL/HA $>$ PCL. The authors concluded that these 3D scaffolds will be applicable for tissue engineering based on unique plotting system.

Chen et al in 2010 ${ }^{17}$ Prepared Composite Electrospun Nanofibers of Polycaprolactone and Nanohydroxyapatite to produce $300 \mathrm{~nm}$ nanofibers containing 0,25 , and $50 \mathrm{wt} \%$ of nHAP for Osteogenic Differentiation of Stem Cells. Nanocomposites of polycaprolactone (PCL) and nanohydroxyapatite (nHAP) were prepared by the composite nanofibers were characterized for structure, morphology, and mechanical properties. Mesenchymal stem cells grown on the nanofibers show different degree of osteogenic differentiation dependent on nHAP content with the highest nHAP content giving the best mineralization.

\section{PCL/BG Composite Membranes}

Fereshteh et al in $2015^{18}$ evaluated Mechanical properties and drug release behavior of $\mathrm{PCL} /$ zein coated 45S5 bioactive glass scaffolds fabricated by foam replication method for bone tissue engineering application. The authors stated that by coating the BG scaffolds with PCL or PCL/zein blend the mechanical properties of the scaffolds were substantially improved, i.e., the compressive strength increased from 0.00470.001 MPa (uncoated BG scaffolds) to 0.1570.02 MPa (PCL/zein coated BG scaffolds). A dense bonelike apatite layer formed on the surface of PCL/zein coated scaffolds immersed for 14 days in simulated body fluid (SBF). The study concluded that the developed scaffolds exhibited attractive properties for application in bone tissue engineering research.

Yufeng Zhang et al in $2014^{19}$ conducted a study aimed to achieve periodontal regeneration of strontiumincorporated mesoporous bioactive glass (Sr-MBG) scaffolds in an osteoporotic animal model carried out by bilateral ovariectomy (OVX). Periodontal fenestration defects treated with Sr-MBG scaffolds showed greater new bone formation $(46.67 \%)$ when compared to MBG scaffolds (39.33\%) and control unfilled samples (17.50\%). The number of TRAP (tartrate-resistant acid phosphatase)-positive osteoclasts was also significantly reduced in defects receiving $\mathrm{Sr}-$ MBG scaffolds. Thus the results suggest that Sr-MBG scaffolds can provide greater periodontal regeneration.

Samaneh Izadi et al in $2014{ }^{20}$ conducted a study to evaluate the nanostructure properties of bioactive glasses. In this research bioglass powder was synthesized with sol-gel method to achieve nanostructure powder. The glass powder was characterized with transmission electron microscope [TEM]. The SEM results show that nanopores and macropores are connectively distributed in whole part of scaffolds. The compressive strength of scaffolds was $0.8 \mathrm{MPa}$. Overall, the scaffold is suggested that is appropriate alternative for bone tissue engineering.
Mansooreh Otadi et al in $2014^{21}$ conducted a study and in that study sol-gel derived glasses based on $\mathrm{CaO}-\mathrm{SrO}-\mathrm{SiO} 2-\mathrm{P} 2 \mathrm{O} 5$ system were prepared. The results showed that the substitution of $\mathrm{Sr}$ for $\mathrm{Ca}$ in the glass, increased the mechanical Strength of nanofibers. composition poly( $\varepsilon$-caprolactone)/bioglass were electrospun using a high DC voltage of $18 \mathrm{kV}$ at a distance of $16 \mathrm{~cm}$. SEM morphology of the PCL/BG electrospun nanocomposite revealed that bioglass nanoparticles were distributed in nanofibers during the electrospinning process. The results revealed that BG contains a higher percentage of strontium oxide increases significantly $(\mathrm{p}<0.05)$ the tensile strength of composite than other BGS.

\section{Electrospinning}

Although numerous membrane materials have been investigated, few studies have focused on the technique of membrane preparation. So far, most GTR membranes are made in the shape of porous form, created by traditional methods such as particulate leaching, solvent casting or gas foaming. Recently, a new technique has been introduced, which is called Electrospinning.

E-spinning is the most widely studied technique and also seems to exhibit the most promising results for TE applications. ${ }^{10}$ The E-spinning technique has demonstrated great potential for processing membranes for periodontal regeneration. E-spinning produces a biocompatible and degradable natural or synthetic polymers that normally resembles the arrangement of the native extracellular matrix (ECM). ${ }^{6}$ Although the concept of E-spinning or electrospraying has been known for more than a century, polymeric nanofibers produced by E-spinning have become a topic of great interest only in the past decade. ${ }^{10}$ Electrospun composite membranes can be tailored with desired new functions by selecting a suitable material or composite and by adjusting the component ratio, fibre diameter and morphology through process parameters. ${ }^{22}$ Electrospinning, allows the preparation of thin fibrous membranes. Electrospinning makes use of a high electric voltage to draw polymer solutions/melts into a whipped jet, which becomes ultrafine fibers after drying in air. Fibers obtained from electrospinning are in the range of $50 \mathrm{~nm}$ to a few microns in diameter and generally collected in the form of a non-woven structure. It has already been shown that electrospun membranes have the potential to promote osteoblastic cell function and bone regeneration. More importantly, the pore size of the electrospun membranes in general is less than the average cell size, and previous studies have shown that such small pores do not allow cell penetration[ Fig. 1]. ${ }^{23}$ 


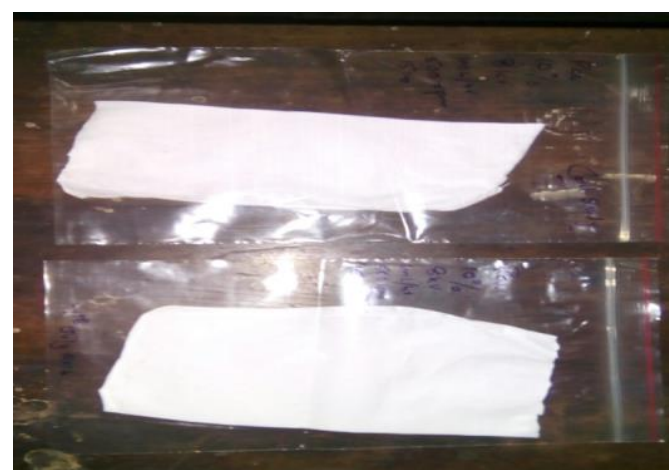

Fig. 1: Electrospun Nanofibrous Sheets

\section{Polycaprolactone[PCL]}

Polycaprolactone (PCL) is a family member of biodegradable aliphatic polyesters [Fig. 2] which have found important use as biomaterials in prosthetics, sutures, and drug delivery systems. As a commercial material, the main attractions of PCL are ${ }^{24}$

1. Its approval by the Food and Drug Administration (FDA) for use in humans,

2. Its biodegradability

3. Its compatibility with a wide range of other polymers

4. Its good processibility which enables fabrication of a variety of structures and forms

5. Its ease of melt processing due to its high thermal stability and

6. Its relatively low cost.

PCL can be easily fabricated into a material possessing the desired toughness. ${ }^{25}$ Overall, PCL has been proven effective for the use in tissue engineering settings. The biocompatibility with the body has been proven. The fact the polymer is bioresorbable helps with numerous tissue engineering factors. With bioresorbable polymers, the fibers provide a back support for the cell growth. After time in the body, the fibers essentially dissolve and leave the cell growth (sometimes in tissue form) in a pure form within the body. Another positive of using polymer, like PCL, is that the body will be more apt to accept the fibers and not cause a potentially devastating immune response cascade. ${ }^{26}$

However, this material alone without additives demonstrates low mechanical resistance to compressive loading, hydrophobicity, and low bioactivity. To counter these problems, Ma et al in 2001 suggested addition of bioactive ceramics to biodegradable polymer composite materials. These new materials demonstrated superior properties including improvement in material strength, stiffness, biodegradability, osteoconductivity, and bioactivity. In addition, polymer/bioactive ceramic composite scaffolds have structures that resemble bone, where the inorganic component of these scaffolds mimics the hydroxycarbonate-apatite (HCA) motifs while the polymer component mimics the collagen-rich extracellular matrix. ${ }^{25}$

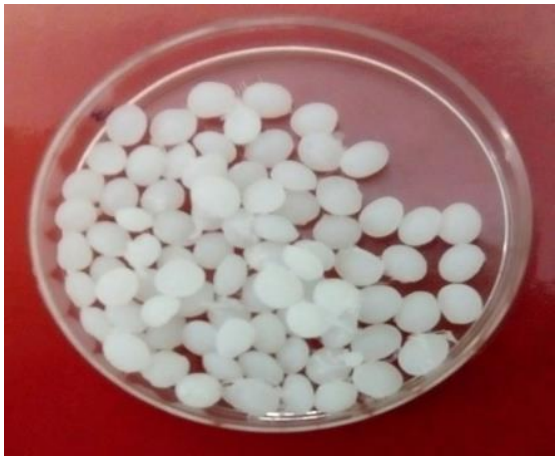

Fig. 2 Polycaprolactone Pellets

\section{Discussion}

Periodontal disease is a chronic inflammatory condition which, if untreated, may ultimately result in tooth loss due to the destruction of the surrounding soft and hard tissues. ${ }^{27,28}$ Periodontal regeneration following surgical treatment requires the reconstitution of the complex structure of the periodontium, which includes formation of periodontal ligament fibres and their insertion into newly formed cementum on the root surface, as well as regeneration of the adjacent alveolar bone. Guided Tissue Regeneration (GTR) (Gottlow et al. 1986, 1990) has emerged as the most widely used regenerative procedure, and relies on the fulfillment of three main principles: wound stabilization, space maintenance and selective cell repopulation of the defect. This technique consists of the placement of an occlusive barrier membrane over the periodontal defect. By this means, cells capable of regenerating the periodontium, namely periodontal ligament cells as well as osteoblasts and their progenitors, are permitted to infiltrate the defect, whereas cell types unable to support regeneration, such as gingival and epithelial cells, are excluded from the regenerating periodontal defect. By selectively allowing competent cells into the defect, GTR-based therapy results in a more effective healing when compared with non-selective procedures, where a poorly or nonorganized collagenous scar tissue is observed, characterized by epithelial down-growth along the root surface which prevents the formation of periodontal attachment. ${ }^{29,30}$

In order for a barrier material to function optimally, it has to meet certain essential design criteria ${ }^{31,32}$

1. Bio-compatibility-The material should not elicit an immune response, sensitization or chronic inflammation which may interfere with healing and present a hazard to the patient.

2. Cell-occlusiveness-The material should act as a barrier to exclude undesirable cell types from entering the secluded space adjacent to the root surface. 
3. Tissue integration- The goal of tissue integration is to prevent rapid epithelial downgrowth on the outer surface of the material or encapsulation of the material, and to provide stability to the overlying flap.

4. Space-making- Barrier material is capable of creating and maintaining a space adjacent to the root surface. This will allow the ingrowth of tissue from the periodontal ligament.

5. Clinical manageability- It should be provided in configurations which are easy to trim and to place.

The barrier membranes used for GTR can be broadly divided into three generations of membranes. The first generation of barrier membranes were non resorbable membranes such as titanium reinforced ePTFE, highdensity-PTFE, or titanium mesh which are aimed to achieve a suitable combination of physical properties to match those of the replaced tissue with a minimal toxic response in the host. The major drawback is the need for second surgery for the removal of the membrane. The second generation of barrier membranes was designed to be resorbable to avoid the need for surgical removal. There are two broad categories of bioresorbable membranes: the natural and the synthetic membranes. Natural membranes are made of collagen or chitosan. Synthetic barrier materials made of polyesters (e.g, poly(glycolic acid) (PGA), poly(lactic acid) (PLA), poly(-caprolactone) (PCL), and their copolymers) were used.

Most of current resorbable synthetic polymer membranes on the market are based on aliphatic polyesters, such as poly(lactic acid) (PLA), poly (glycolic acid) (PGA), poly ( $\varepsilon$-caprolactone) (PCL), poly (hydroxyl valeric acid), and poly (hydroxyl butyric acid), as well as their copolymers. Due to its biocompatibility, low cost and high mechanical strength, polycaprolactone (PCL) is an attractive biomedical polymer and has been extensively studied in bone tissue engineering. PCL does not produce a local acidic environment during the degradation procedure compared with PLA and PLGA. ${ }^{33,34}$

One of the limitations of membranes fabricated with PCL is their poor cell-scaffold interactions due to the inherent hydrophobic nature. This may lead to poor cell adhesion, migration, proliferation, and differentiation during cell culture. Hence strategies to improve the hydrophilicity of PCL based scaffolds are essential. To overcome these problems, recent research efforts have included the incorporation of bone-like ceramics into the membranes, e.g. hydroxyapatite, tricalcium phosphate and calcium carbonate. In these efforts, nano-sized ceramic particles are of particular interest as they mimic the mineral crystals as present in the natural tissue and have been shown to induce a significant increase in protein absorption and cell adhesion, compared to their micro-sized counterparts. ${ }^{35}$ Although numerous membrane materials have been investigated, few studies have focused on the technique of membrane preparation. So far, most GTR/GBR membranes are made in the shape of porous foam, created by traditional methods such as particulate leaching, solvent casting or gas foaming. ${ }^{36,14}$

Recently, a new technique has been introduced, called electrospinning, which allows the preparation of thin fibrous membranes. ${ }^{36}$ Electrospinning, a spinning technique, is a unique approach using electrostatic forces to produce fine fibers from polymer solutions or melts and the fibers thus produced have a thinner diameter (from nanometer to micrometer) and a larger surface area than those obtained from conventional spinning processes. Furthermore, a DC voltage in the range of several tens of Kvs is necessary to generate the electrospinning. This process, mainly based on the principle that strong mutual electrical repulsive forces overcome weaker forces of surface tension in the charged polymer liquid (Chew et al., 2006) [Fig. $3]$. Currently, there are two standard electrospinning setups, vertical and horizontal. With the expansion of this technology, several research groups have developed more sophisticated systems that can fabricate more complex nanofibrous structures in a more controlled and efficient manner (Kidoaki et al., 2005; Stankus et al., 2006). ${ }^{36-38}$ Electrospinning is conducted at room temperature with atmosphere conditions. Basically, an electrospinning system consists of three major components: a high voltage power supply, a spinneret (e.g., a pipette tip) and a grounded collecting plate (usually a metal screen, plate, or rotating mandrel) and utilizes a high voltage source to inject charge of a certain polarity into a polymer solution or melt, which is then accelerated towards a collector of opposite polarity (Liang et al., 2007; Sill and Recum, 2008). Most of the polymers are dissolved in some solvents before electrospinning, and when it completely dissolves, forms polymer solution. The polymer fluid is then introduced into the capillary tube for electrospinning. ${ }^{39-41}$ In the electrospinning process, a polymer solution held by its surface tension at the end of a capillary tube is subjected to an electric field and an electric charge is induced on the liquid surface due to this electric field. When the electric field applied reaches a critical value, the repulsive electrical forces overcome the surface tension forces. Eventually, a charged jet of the solution is ejected from the tip of the Taylor cone and an unstable and a rapid whipping of the jet occurs in the space between the capillary tip and collector which leads to evaporation of the solvent, leaving a polymer behind. (Taylor, 1969, Yarin et al., 2001; Adomaviciute and Milasius, 2007). The jet is only stable at the tip of the spinneret and after that instability starts. ${ }^{36,42-45}$ Thus, the electrospinning process offers a simplified technique for fiber formation. Fibers obtained from electrospinning are in the range of $50 \mathrm{~nm}$ to a few microns in diameter and generally collected in the form of a non-woven structure. It has already been shown that electrospun 
membranes have the potential to promote osteoblastic cell function and bone regeneration. ${ }^{35}$

Electrospinning technique is governed by various processing parameters such as solution viscosity, applied potential, flow rate, tip to collector distance, solvent nature, needle diameter and various ambient parameters. Electrospinning helps in obtaining bead free nano fibers. It is observed that blending PCL with different concentrations of bioactive materials resulted in smooth fibers with reduced fiber diameter. This reduction in fiber diameter can be attributed to difference in solution viscosity i.e when viscosity decreases conductivity increases and this conductivity is inversely proportional to fiber diameter.Fiber diameter can also be reduced with the incorporation of nHAP. calcium and phosphate ions in nHAP which provides higher conductivity leads to decreased viscosity. ${ }^{14}$

Pore diameter and fiber diameter plays a vital role in the biological performance of scaffold as it determines both cell-cell as well as cell- membrane interaction. High porosity, adequate pore size and interconnected pore network are essential criteria for a tissue engineering as it enables better cell infiltration and vascularization. ${ }^{14,46}$ The reduction in pore size occurs as more layers of fibers might overlap with each other, especially when the fiber diameter is smaller, resulting in smaller pore diameter. The pore size distribution lies in a range below $300 \mu \mathrm{m}$ for all the scaffolds. The preferable pore size for osteoblast cells ranges from 200 to $400 \mu \mathrm{m}$ for encouraging migration, attachment and proliferation. However for electrospun matrices pores formed are much smaller than the normal cell size of a few to tens of micrometer. Pores in an electrospun structure are formed by the randomly oriented fibers lying loosely upon each other. Cells can migrate through pores by their amoeboid movement and can push surrounding fibers aside to expand the hole. This dynamic architecture of fibers allows cells to adjust according to pore size and grow into nanofiber matrices. ${ }^{14}$ Regarding the mechanical properties, the membranes fabrticated by electrospinning techniques exhibits good Tensile strength, Youngs modulus, and elongation at break. The mechanical properties as well as the chemical properties are far more superior than membranes fabricated by techniques other than electrospinning.

Apart from favorable physicochemical and mechanical properties, the most important requirement for a biomaterial is its biocompatibility in a specific environment, together with the non cytotoxicity of its degradation products. ${ }^{47}$ Ogawa et al in $2016^{48}$ stated that, nano-modification of biomaterials might increase the surface area and adsorption of signaling molecules. The observation from the above mentioned study confirms the bioactivity of the electrospinned PCL membranes reinforced with BG/Nhap and its usefulness in periodontal tissue engineering as a GTR/GBR membrane for proliferation and differentiation in to specific cell lineage.

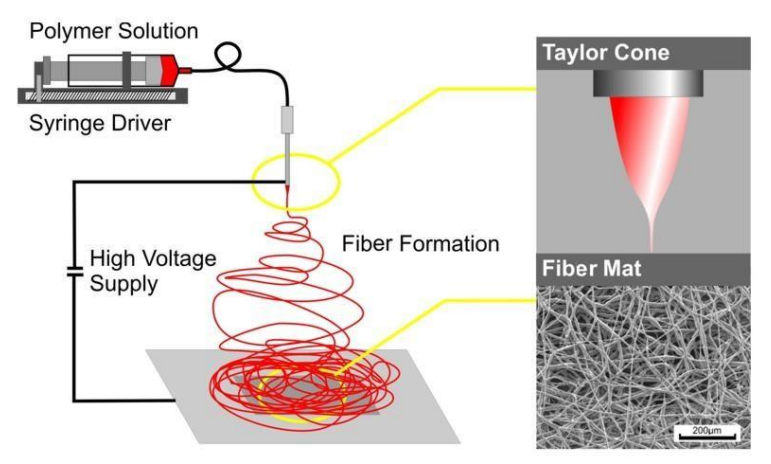

Fig 3: Electrospinning Technique

[http://loadvenue.com/electro-optical-

instrumentation-sensing-and-measuring-with-laserssilvano-donati-pdf-9563]

\section{Conclusion}

The optimal resorbable membrane for GTR or GBR has to be strong, able to stimulate bone formation and promote osteoblast-like cell proliferation and differentiation. The composite nanofibrous membranes nHAP/BG and PCL prepared by electrospinning will exhibit superior physiochemical, mechanical and in vitro properties. However, the membrane with a high nHAP/BG loading density was weaker than the one with low nHAP/BG loading density. Based on numerous clinical and non clinical study results, we conclude that the composite nanofibrous membrane prepared by Electrospinning method are far superior to membranes fabricated by other techniques and hence an ideal material to be used as a GTR membrane/GBR membrane for periodontal regeneration.

\section{References}

1. Vargas S, Ilyina A, Segura C, Silva B, M eacute ndez G. Etiology and microbiology of periodontal diseases: A review. Afr J Microbiol Res 2015;9(48):2300-6.

2. Laurell L, Gottlow J. Guided tissue regeneration update. Inter Dent J 1998;48(4):386-98.

3. Position Paper: Periodontal Regeneration. J Periodontol 2005;76(9):1601-22.

4. Shimauchi H, Nemoto E, Ishihata H, Shimomura M. Possible functional scaffolds for periodontal regeneration. Jpn Dent Sci Review 2013;49(4):118-30.

5. Singh AK. GTR membranes: The barriers for periodontal regeneration. DHR-IJMS 2013;4(1):31-8.

6. Sam G. Evolution of Barrier Membranes in Periodontal Regeneration-“Are the third Generation Membranes really here?". J Clin Diagn Res. 2014;ZE14-ZE17

7. Cannillo V, Fabbri P, Sola A. Fabrication of 45S5 bioactive glass- polycaprolactone composite scaffolds. ICCM Int Conf Compos Mater [Internet]. 2009.

8. Guo B, Lei B, Li P, Ma P. Functionalized scaffolds to enhance tissue regeneration. Regen Biomater 2015;2(1):47-57. 
9. Prakash Mb, Kumar Nag M, Mohammed A, Bhargavi K, Sai Ram M, Rajkumar R. Poly ( $\varepsilon$-caprolactone) PCL Scaffolds Preparation and characterization for tissue engineering. Int J Mech Eng Robot 2014;1(2):2321-5747.

10. Vasita R, Katti DS. Nanofibers and their applications in tissue engineering. Int J Nanomedicine 2006;1(1):15-30.

11. Boccaccini AR, Chatzistavrou X, Yunos DM, Califano V. Biodegradable Polymer-Bioceramic Composite Scaffolds for Bone Tissue Engineering. Proc $17^{\text {th }}$ Int Conf Compos Mater. 2009.

12. Aurer A, JorgiE-Srdjak K. Membranes for Periodontal Regeneration. Acta Stomatol Croat 2005;39(1):107-12.

13. Battistella E, Varoni E, Cochis A, Palazzo B, Rimondini L. Degradable polymers may improve dental practice. $\mathrm{J}$ Appl Biomater Biomech 2011;9(3):223-31.

14. Remya KR, Joseph J, Mani S, John A, Varma HK, Ramesh P. Nanohydroxyapatite incorporated electrospun polycaprolactone/ polycaprolactone- polyethyleneglycolpolycaprolactone blend scaffold for bone tissue engineering applications. J Biomed Nanotechnol 2013;9(9):1483-94.

15. Hassan MI, Mokhtar M, Sultana N, Khan TH. Production of Hydroxyapatite (HA) nanoparticle and HA / PCL Tissue Engineering Scaffolds for Bone Tissue Engineering. IECBES 2012;239-42.

16. Park SA, Hee S, Wan L, Kim D. Fabrication of porous polycaprolactone / hydroxyapatite (PCL / HA) blend scaffolds using a 3D plotting system for bone tissue engineering. Bioprocess Biosyst Eng 2011;34(4):505-13.

17. Chen J, Lai G, Chang Y. Preparation of Composite Electrospun Nanofibers of Polycaprolactone and Nanohydroxyapatite for Osteogenic Differentiation of Stem Cells. Nanoelectronics Conference (INEC), 2010.

18. Fereshteh Z, Nooeaid P, Fathi M, Bagri A, Boccaccini AR. Mechanical properties and drug release behavior of PCL/zein coated 45S5 bioactive glass scaffolds for bone tissue engineering application. Data $\mathrm{Br}$ [Internet]. Elsevier; 2015;4:524-8.

19. Zhang Y, Wei L, Wu C, Miron RJ. Periodontal regeneration using strontium-loaded mesoporous bioactive glass scaffolds in osteoporotic rats. PLoS One. 2014;9(8):1-6.

20. Izadi S, Hesaraki S, Hafezi-Ardakani M. Evaluation Nanostructure Properties of Bioactive Glass Scaffolds for Bone Tissue Engineering. Adv Mater Res [Internet]. 2013; 829:289-93.

21. Nanocomposites E, Otadi M, Mohebbi D. The Effect of Bioglass Nanoparticles and Strontium Component on Tensile Strength of Polycaprolactone / Bioglass. 2014;23-4.

22. Balu R, Singaravelu S, Nagiah N. Bioceramic Nanofibres by Electrospinning. Fibers. 2014;221-39.

23. Yang F, Both SK, Yang X, Walboomers XF, Jansen JA. Development of an electrospun nano-apatite/PCL composite membrane for GTR/GBR application. Acta Biomater [Internet]. Acta Materialia Inc.; 2009;5(9):3295-304.

24. Azimi B, Nourpanah P, Rabiee M, Arbab S. Poly (lactide -co- glycolide) Fiber: An Overview. J Eng Fiber Fabr [Internet] 2014;9(3):74-90.

25. Naghizadeh F, Sultana N, Abdul Kadir MR, Tengku Md Shihabudin TM, Hussain R, Kamarul T. The fabrication and characterization of PCL/rice husk derived bioactive glass-ceramic composite scaffolds. J Nanomater 2014;2014.p.9.

26. Montero GA, Gluck JM, King MW. Polycaprolactone (Pcl) Nonwoven Mats for Use as Tissue Engineering Scaffolds Generate by Electrospinning Process.
Electrospinning process College of Textiles, North Carolina State University, Raleigh.

27. Newman MG, Takei HH, Klokkevold PR, Carranze FA Carranza's Clinical Periodontology. $11^{\text {th }}$ Edition. India: Elsevier Saunders; 2012.

28. Vargas SAI, Ilyina A, Segura CEP, Silva BY, et al. Etiology and microbiology of periodontal diseases: A review. African J Microbiol Res. 2015;9(48):2300-6.

29. Regeneration P. Academy Report 2005;1601-22.

30. Costa PF, Vaquette C, Zhang Q, Reis RL, Ivanovski S, Hutmacher DW. Advanced tissue engineering scaffold design for regeneration of the complex hierarchical periodontal structure. J Clin Periodontol 2014;41(3):28394.

31. Laurell L, Gottlow J. Guided tissue regeneration update. Int Dent J 1998;48(1 998):386-98.

32. Aurer A, JorgiE-Srdjak K. Membranes for Periodontal Regeneration. Acta Stomatol Croat 2005;39(1):107-12.

33. Chen F-M, Jin Y. Periodontal tissue engineering and regeneration: current approaches and expanding opportunities. Tissue Eng Part B Rev 2010;16(2):219-55.

34. Wang J, Wang L, Zhou Z, Lai H, Xu P, Liao L, et al. Biodegradable polymer membranes applied in guided bone/tissue regeneration: A review. Polymers 2016;8(4):115.

35. Abou Neel EA, Chrzanowski W, Salih VM, Kim HW, Knowles JC. Tissue engineering in dentistry. J Dent 2014;42(8):915-28.

36. Yang F, Both SK, Yang X, Walboomers XF, Jansen JA. Development of an electrospun nano-apatite/PCL composite membrane for GTR/GBR application. Acta Biomater 2009;5(9):3295-304.

37. Bhardwaj N, Kundu SC. Electrospinning: A fascinating fiber fabrication technique. Biotechnol Adv 2010;28(3):325-47.

38. Agarwal S, Wendorff JH, Greiner A. Use of electrospinning technique for biomedical applications. Polymer (Guildf) 2008;49(26):5603-21.

39. Vonch J, Yarin a, Megaridis CM. Electrospinning: A study in the formation of nanofibers. J Undergrad Res 2007;1(1):1.

40. Chen H, Blitterswijk CVAN, Moroni L. Fabrication of nanofibrous scaffolds for tissue engineering applications. Woodhead Publ Online. 2013.

41. Zhang Y, Su B, Venugopal J, Ramakrishna S, Lim C. Biomimetic and bioactive nano fi brous scaffolds from electrospun composite nano fibers. Int J Nanomedicine 2007;1(9):623-38.

42. Goh Y-F, Shakir I, Hussain R. Electrospun fibers for tissue engineering, drug delivery, and wound dressing. J Mater Sci 2013;48(8):3027-54.

43. Pham QP, Sharma U, Mikos AG. Electrospinning of polymeric nanofibers for tissue engineering applications: a review. Tissue Eng. 2006;12(5):1197-211.

44. Shin S-H, Purevdorj O, Castano O, Planell J a, Kim H-W. A short review: Recent advances in electrospinning for bone tissue regeneration. J Tissue Eng. 2012;3(1):2041731412443530.

45. Hasan MM, Alam AKMM, Nayem KA. Application of Electrospinning Techniques for the Production of Tissue Engineering Scaffolds: a Review. Eur Sci J. 2014;10(15):1857-7881.

46. Athira KS, Sanpui P, Chatterjee K. Fabrication of Poly(Caprolactone) Nanofibers by Electrospinning. J Polym Biopolym Phys Chem 2014;2(4):62-6.

47. Joseph D. Evaluating the Potential of Electrospun Membranes for Tissue Specific Signal Delivery in Cartilage and Bone Tissue Engineering. 2013. 
48. Ogawa K, Miyaji H, Kato A, Kosen Y, Momose T, Yoshida T, et al. Periodontal tissue engineering by nano beta-tricalcium phosphate scaffold and fibroblast growth factor- 2 in one-wall infrabony defects of dogs. J Periodontal Res. 2016;51(6):75867. 